\title{
Where Does the First Lateral Pelvic Lymph Node Receive Drainage from?
}

\author{
Hidejiro Kawahara Hiroshi Nimura Kazuhiro Watanabe Tetsuya Kobayashi \\ Hideyuki Kashiwagi Katsuhiko Yanaga \\ Department of Surgery, Jikei University School of Medicine, Tokyo, Japan
}

\section{Key Words}

Rectal cancer - Lateral pelvic lymph node metastasis .

Lateral pelvic lymph node dissection • Infrared ray

electronic endoscopy

\begin{abstract}
Background: Lateral pelvic lymph node dissection (LPLD) in the treatment of rectal cancer has risks and benefits. Avoidance of unnecessary LPLD is important, however, preoperative and/or intraoperative accurate detection of lateral lymph node metastases have not been established. If the lateral lymph node to which the fluid first spread from the primary lower rectal cancer is detected accurately, it may guide the need for LPLD and may assist in avoiding unnecessary dissection. Methods: A total of 14 patients with T3 lower rectal cancer were evaluated to locate the lymph nodes through which indocyanine green (ICG) reached the lymphatics. After ICG was injected into the lower rectum via an endoscope preoperatively, total mesorectal excision was first performed, and LPLD was performed with infrared ray electronic endoscopy (IREE) to assess the degree of retention of ICG in each regional lymph node. Results: Drainage of ICG to lateral pelvic lymph nodes was observed in 6 of 14 patients (43\%). All ICG-containing lymph nodes were detected by IREE. When present, lateral pelvic wall lymph node
\end{abstract}

drainage was limited exclusively to the peri-internal iliac artery nodes. No obturator nodes were involved. Conclusion: The first lateral lymph node that receives lymphatic drainage from lower rectal cancer is located around the internal iliac arteries.

Copyright $\odot 2007$ S. Karger AG, Basel

\section{Introduction}

Anatomic studies on lymphatic drainage of the rectum have been conducted by injecting a dye into cadavers. Gerota [1] first described lateral and upward lymphatic flow of the rectum in 1895 with such a technique. In Japan, Senba [2] conducted an anatomic study on lymphatics of the rectum by injecting a dye into cadaver fetuses and concluded that the lateral lymphatic vessels are distributed around the internal iliac arteries and inside the obturator spaces.

The clinical importance of the lateral spread of lower rectal cancer was first demonstrated in the 1950s [3]. Based on these reports, both total mesorectal excision (TME) and lateral pelvic lymph node dissection (LPLD) had been performed for advanced rectal cancer in Japan, which has been reported to reduce local recurrence as compared to conventional surgery [4]. On the contrary,

\section{KARGER}

Fax +4161306 1234

E-Mail karger@karger.ch

www.karger.com
(C) 2007 S. Karger AG, Basel

0253-4886/07/0246-0413\$23.50/0

Accessible online at:

www.karger.com/dsu
Hidejiro Kawahara, MD, PhD

Department of Surgery, Jikei University School of Medicine

Nishi-Shinbashi 3-25-8, Minato-ku, Tokyo 105-8461 (Japan)

Tel. +81 33433 1111, ext. 3401, Fax +81 334358677

E-Mail kawahide@jikei.ac.jp 
Table 1. Clinicopathological features of the patients who underwent curative resection for $\mathrm{T} 3$ lower rectal cancer

\begin{tabular}{llc}
\hline Variable & & Patients \\
\hline Gender & Male & $9(64 \%)$ \\
& Female & $5(36 \%)$ \\
Age, years (range) & & $57.6(37-75)$ \\
Tumor diameter, mm (range) & & $53.1(30-70)$ \\
Histological findings & Well & $5(36 \%)$ \\
& Moderate & $9(65 \%)$ \\
Lymphatic invasion & Yes & $14(100 \%)$ \\
& No & $0(0 \%)$ \\
Venous invasion & Yes & $13(93 \%)$ \\
& No & $1(7 \%)$ \\
Lymph node metastasis & Yes & $13(93 \%)$ \\
& No & $1(7 \%)$ \\
Lateral node metastasis & Yes & $4(29 \%)$ \\
& No & $10(71 \%)$ \\
\hline
\end{tabular}

Well = Well-differentiated adenocarcinoma; Moderate = moderately-differentiated adenocarcinoma.

LPLD in the treatment of rectal cancer increases the incidence of urinary and male sexual dysfunction by injury to the autonomic nerve in the pelvis. LPLD has advantages and disadvantages related to local recurrence and survival. In advanced rectal cancer, the incidence of lateral pelvic lymph nodes metastases had been reported to be $15-20 \%$ [5]. However, the first lateral pelvic lymph node that encounters cancer cells from rectal cancers is yet to be clarified. Accurate identification of such a 'sentinel' lymph node and its metastatic status may abrogate unnecessary LPLD. In this study, we tried to identify the initial lateral pelvic lymph node for lower rectal cancer.

\section{Patients and Methods}

The protocol was approved by the Ethics Committee for Biomedical Research of the Jikei Institutional Review Board for a 2year period between 2003 and 2004, and all patients provided informed consent. Patients admitted to Jikei University Kashiwa Hospital with T3 lower rectal cancer were enrolled prospectively in the study.

A total of 14 patients with T3 lower rectal cancer were included in the study. Their mean age was 57.6 years, with a range of $37-75$ years, and 5 of them were female. Before surgery in the operation room, approximately $6 \mathrm{ml}$ of indocyanine green (ICG; $5 \mathrm{mg} / \mathrm{ml}$ ) was injected in the submucosal tissue at three sites: the right, left and anal sides of the tumor using an endoscopic puncture needle.

Thirty minutes after the injection, open surgery was started. Following TME, lateral pelvic dissection was performed under
Table 2. The status of lymph node involvement

\begin{tabular}{|c|c|c|c|c|c|c|}
\hline \multirow{2}{*}{$\begin{array}{l}\text { Patient } \\
\text { No. }\end{array}$} & \multicolumn{2}{|c|}{ Upward nodes } & \multicolumn{2}{|c|}{ Lateral pelvic nodes } & \multicolumn{2}{|l|}{ Total } \\
\hline & positive & total & positive & total & positive & total \\
\hline 1 & 3 & 11 & 0 & 18 & 3 & 29 \\
\hline 2 & 1 & 12 & 0 & 17 & 1 & 29 \\
\hline 3 & 5 & 16 & 0 & 16 & 5 & 32 \\
\hline 4 & 0 & 11 & 0 & 20 & 0 & 31 \\
\hline 5 & 2 & 17 & 0 & 19 & 2 & 36 \\
\hline 6 & 3 & 24 & 0 & 15 & 3 & 39 \\
\hline 7 & 5 & 13 & 0 & 19 & 5 & 32 \\
\hline 8 & 2 & 14 & 0 & 14 & 2 & 28 \\
\hline 9 & 3 & 11 & 0 & 17 & 3 & 28 \\
\hline 10 & 2 & 15 & 0 & 15 & 2 & 30 \\
\hline 11 & 5 & 16 & 2 & 17 & 7 & 33 \\
\hline 12 & 4 & 23 & 1 & 16 & 5 & 39 \\
\hline 13 & 4 & 12 & 1 & 16 & 5 & 28 \\
\hline 14 & 2 & 12 & 2 & 18 & 4 & 30 \\
\hline Mean & 2.9 & 14.8 & 0.4 & 16.9 & 3.4 & 31.7 \\
\hline Range & $0-5$ & $11-24$ & $0-2$ & $14-20$ & $0-7$ & $28-39$ \\
\hline
\end{tabular}

the guidance of infrared ray electronic endoscopy (IREE; Olympus Optical, Tokyo, Japan) to illuminate bilateral pelvic regional lymph nodes.

\section{Results}

Clinicopathological Features of 14 Patients (table 1)

We analyzed the medical records of the 14 patients. All patients presented with adenocarcinoma, and lymph node metastasis was positive in 13 patients $(93 \%)$ including 4 in the lateral nodes.

Correlation between Number of Positive Lymph Nodes and Dissected Lymph Nodes (table 2)

The mean number of upward lymph nodes dissected was 14.8 , with a range of 11-24, including a mean of 2.9 cancer-positive lymph nodes. The mean number of lateral lymph nodes dissected was 16.9, with a range of 1420 , including a mean of 0.4 cancer-positive lymph nodes. The total mean number of lymph nodes dissected was 31.7 , with a range of $28-39$, including a mean of 3.4 cancer-positive lymph nodes.

\section{ICG Detectability of Lymph Nodes (table 3)}

There were no adverse events after injection of ICG. All IREE-positive upward lymph nodes were detected macroscopically. However, not all IREE-positive lateral 

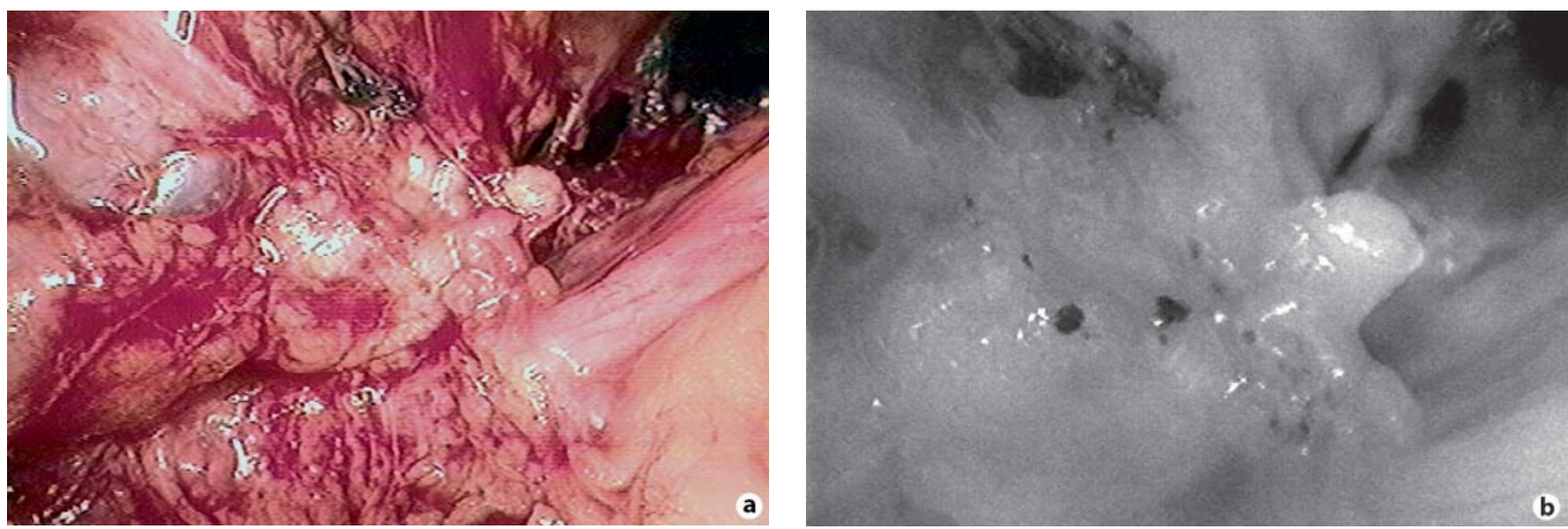

Fig. 1. Dissection of lymph nodes around the left internal iliac artery $90 \mathrm{~min}$ after injection of ICG in the lower rectum. a Lymph node staining with ICG alone is not clear to the naked eye. $\mathbf{b}$ The same area observed under illumination with IREE. Lymph nodes are identified clearly.

Table 3. Relationship between the number of positive lymph nodes and dissected lymph nodes in lateral pelvis

\begin{tabular}{|c|c|c|c|c|c|c|c|c|c|c|}
\hline \multirow{3}{*}{$\begin{array}{l}\text { Patient } \\
\text { No. }\end{array}$} & \multicolumn{10}{|c|}{ Lateral pelvic nodes } \\
\hline & \multicolumn{2}{|l|}{ total } & \multicolumn{2}{|c|}{ right peri-iliac artery } & \multicolumn{2}{|c|}{ right obturator space } & \multicolumn{2}{|c|}{ left peri-iliac artery } & \multicolumn{2}{|c|}{ left obturator space } \\
\hline & positive & total & positive & total & positive & total & positive & total & positive & total \\
\hline 1 & 0 & 18 & 0 & 3 & 0 & 6 & 0 & 3 & 0 & 6 \\
\hline 2 & 0 & 17 & 0 & 3 & 0 & 5 & 0 & 4 & 0 & 5 \\
\hline 3 & 0 & 16 & 0 & 2 & 0 & 6 & 0 & 2 & 0 & 6 \\
\hline 4 & 0 & 20 & 0 & 4 & 0 & 7 & 0 & 4 & 0 & 5 \\
\hline 5 & 0 & 19 & 0 & 4 & 0 & 5 & 0 & 4 & 0 & 6 \\
\hline 6 & 0 & 15 & 0 & 2 & 0 & 5 & 0 & 3 & 0 & 5 \\
\hline 7 & 0 & 19 & 0 & 2 & 0 & 6 & 0 & 3 & 0 & 8 \\
\hline 8 & 0 & 14 & 0 & 2 & 0 & 5 & 0 & 2 & 0 & 5 \\
\hline 9 & 0 & 17 & 0 & $2^{1}$ & 0 & 6 & 0 & $3^{1}$ & 0 & 6 \\
\hline 10 & 0 & 15 & 0 & $2^{1}$ & 0 & 5 & 0 & $2^{1}$ & 0 & 6 \\
\hline 11 & 2 & 17 & 1 & $3^{1}$ & 0 & 6 & 1 & $3^{1}$ & 0 & 5 \\
\hline 12 & 1 & 16 & 0 & $2^{1}$ & 0 & 5 & 1 & $3^{1}$ & 0 & 6 \\
\hline 13 & 1 & 16 & 0 & $3^{1}$ & 0 & 5 & 1 & $3^{1}$ & 0 & 5 \\
\hline 14 & 2 & 18 & 1 & $3^{1}$ & 0 & 6 & 1 & $3^{1}$ & 0 & 6 \\
\hline Mean & 0.4 & 16.9 & 0.1 & 2.6 & 0 & 5.6 & 0.3 & 3.1 & 0 & 5.7 \\
\hline Range & $0-2$ & $14-20$ & $0-1$ & $2-4$ & 0 & $5-7$ & $0-1$ & $2-4$ & 0 & $5-8$ \\
\hline
\end{tabular}

${ }^{1}$ Lateral lymph nodes are detected by ICG with infrared ray electronic endoscopy.

lymph nodes were detected macroscopically. IREE was very useful in detecting regional lymph nodes with a very small amount of ICG (fig. 1, 2). ICG in lateral lymph nodes was observed in only 6 patients (43\%) including all 4 patients with lateral lymph node metastasis. Metastatic lateral lymph nodes were detected only in bilateral lymph nodes around the internal iliac arteries. On the other hand, no positive lymph nodes were detected in the obturator spaces bilaterally. ICG detected in lateral lymph nodes were located around both internal iliac arteries only, and not in either obturator spaces. 
Fig. 2. Dissected lymph node from the peri-internal iliac artery space. a Lymph node staining with ICG alone is not clear to the naked eye. $\mathbf{b}$ The same area observed under illumination with IREE demonstrating the lymph node to be positive.
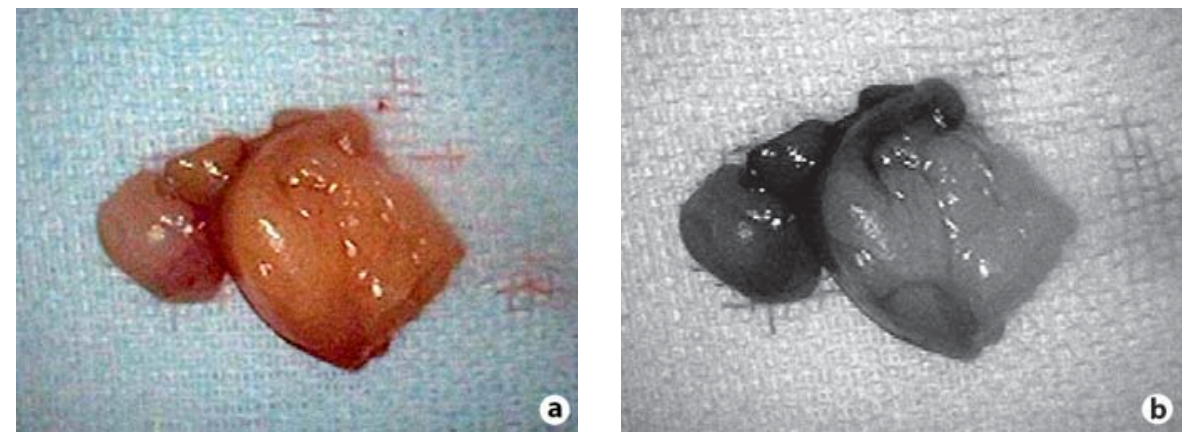

\section{Discussion}

As for the surgical procedure for advanced rectal cancer, TME [6] is the gold standard in western countries. In Japan, LPLD had been performed to improve prognosis since the 1930s [7]. In spite of a 15-20\% [5] incidence of lateral lymph node metastases in advanced rectal cancer, accurate detection of lateral lymph node metastases before or during an operation is yet to be established. For breast cancer $[8,9]$ and gastric cancer $[10,11]$, the concept of sentinel lymph node has been clinically adopted to avoid unnecessary radical lymph nodes dissections. The sentinel lymph node is defined as the first lymph node that drains lymphatic fluid into or from the primary cancer site. If the lateral lymph node to which the fluid first spread from the primary lower rectal cancer is detected accurately, it may guide the need for LPLD.

For gastric cancer, physiological lymph flow can be visualized by ICG injection [12] from the injection site to the regional lymph nodes in real time. However, rectal lymph flow cannot be observed directly because all regional lymph nodes and lymphatic vessels are located in the retroperitoneum. Therefore, we tried to evaluate the location of the lateral lymph nodes that drain first the lower rectal cancer using ICG that flows through the lymphatic system in the same duration after the injection of ICG, approximately $90 \mathrm{~min}$. The lymph flow drainage toward lateral lymph nodes was intercepted by TME, and we started to evaluate the lateral lymph nodes at approximately 90 min after the injection of ICG.

All lateral lymph nodes draining ICG were located around the internal iliac artery, and lateral lymph node metastases were detected only around the internal iliac arteries of all 4 such patients. These findings strongly support that the sentinel lateral lymph node, to which the lymph fluid first spreads from the lower rectal cancer, is located around the internal iliac arteries.
Senba [2] described that lymphatic drainage toward the lateral pelvis was bidirectional, i.e., toward internal iliac arteries and obturator spaces. In this study, drainage of ICG could not be observed in the lymph nodes of the obturator spaces. Therefore, it is suggested that lymph flow from the lower rectum toward the lymph nodes around the internal iliac arteries is significantly greater than that toward the obturator spaces.

As to the relationship between lymph flow toward upward lymph nodes and lateral ones, the drainage of ICG toward upward lymph nodes was confirmed macroscopically. However, the drainage of ICG toward lateral lymph nodes could not be detected without IREE. This suggested that lymph flow from the lower rectum is mainly upward and less toward the lateral pelvis. Quantity and speed of lymph flow seems to be related to the incidence of lymph node metastases.

\section{Conclusion}

We have demonstrated using IREE with ICG that the first lateral lymph node that receives drainage from lower rectal cancer is located around the internal iliac arteries.
References
1 Gerota D: Die Lymphgefaesse des Rectums und Anus. Arch Anat Physiol Anat Abt 1895; 240-256.

2 Senba Y: An anatomical study of lymphatic system of the rectum (in Japanese). J Hukuoka Med Coll 1927;20:1213-1268.

-3 Saucer I, Bacon HE: Influence of lateral spread of cancer of the rectum on radicability of operation and prognosis. Am J Surg 1951;81:111-120. 
4 Moriya Y, Sugihara K, Akasu T, Fujita S: Patterns of recurrence after nerve-sparing surgery for rectal adenocarcinoma with special reference to loco-regional recurrence. Dis Colon Rectum 1995;38:1162-1168.

5 Takahashi T, Ueno M, Azekura K, Ohta H: Lateral node dissection and total mesorectal excision for rectal cancer. Dis Colon Rectum 2000;43(suppl):S59-S68.

6 Heald RJ, Husband EM, Ryall RD: The mesorectum in rectal cancer surgery - the clue to pelvic recurrence? Br J Surg 1982;69:613616.
7 Kuru M: Cancer of the rectum (in Japanese). J Jpn Surg Soc 1940;41:832-877.

-8 Veronesi U, Paganelli G, Galimberti V, Viale G, Zurrida S, Bedoni M, et al: Sentinel-node biopsy to avoid axillary dissection in breast cancer with clinically negative lymph nodes. Lancet 1997;349:1864-1867.

-9 Schrenk P, Wayand W: Sentinel-node biopsy in axillary lymph-node staging for patients with multicentric breast cancer. Lancet 2001; 357:122.
10 Miwa K, Kinami S, Taniguchi K, Fushida S, Fujimura T, Nonomura A: Mapping sentinel nodes in patients with early-stage gastric carcinoma. Br J Surg 2003;90:178-182.

11 Kitagawa Y, Fujii H, Mukai M, Kubota T, Otani Y, Kitajima M: Radio-guided sentinel node detection for gastric cancer. Br J Surg 2002;89:604-608.

2 Ninura H, Narimiya N, Mitsumori Y, Yamazaki Y, Yanaga K, Urashima M: Infrared ray electronic endoscopy combined with indocyanine green injection for detection of sentinel nodes of patients with gastric cancer. Br J Surg 2004;91:575-579. 